\title{
Giant breast tumour in a 13-year-old girl
}

\section{Barbara Madej', Mateusz Szymański², Wojciech Dworzański², Ewa Dudek², Maciej Czerwonka², Wojciech Kwaśniewski ${ }^{3}$}

\author{
${ }^{1}$ Breast Surgery Department, the Cardinal Rev. Wyszynski Voivodship Specialist Hospital in Lublin, Poland \\ ${ }^{2}$ Anatomy Department, Medical University of Lublin, Poland \\ 3 1st Department and Clinic of Gynecologic Oncology and Gynecology, Medical University of Lublin, Poland
}

Madej B, Szymański M, Dworzański W, Dudek E, Czerwonka M, Kwaśniewski W. Giant breast tumour in a 13-year-old girl. Ann Agric Environ Med. 2017; 24(3): 541-543. doi: 10.5604/12321966.1228396

\section{Abstract}

This study describes a rare case of a giant phyllodes tumor in a 13-year-old girl. The authors have conducted an analysis of the diagnostic process and have shown the results of operative treatment of the tumor. Moreover, organisational aspects of the diagnostics concerning breast diseases in patients from smaller towns in Poland have been discussed. It has been indicated that the availability of suitable diagnostics and treatment of breast diseases in children and teenagers from rural areas and smaller towns is limited.

\section{Key words}

Tumor phyllodes, Breast, children

\section{INTRODUCTION}

Phyllodestumour is a breast neoplasm that usually occurs in patients aged $30-70[1,2]$. It affects about $0.3-1 \%$ of women with diagnosed breast neoplasms $[3,4,5,6]$. The most frequently noted size of phyllodestumour is $2-7 \mathrm{~cm}$, yet there have often been cases of lesions reaching even $20-30 \mathrm{~cm}$. In some patients the lesion fills the whole breast leading to the destruction of the gland and skin $[7,8]$.

Histologically, the tumour is formed from stroma of connective tissue and epithelial elements $[7,4]$. In rare cases it can be a component of ductal cancer [8]. According to the WHO classification there are 3 types of phyllodestumours: low, medium and high malignant potential [9]. Low malignancy tumours are characterised by fast local growth without tendency to form distant metastases. However, even in such cases there is a significant risk of recurrence. Local recurrence affecting all types of phyllodestumour is estimated at $8-36 \%[4,9]$. For medium malignant potential lesions the risk of recurrence is $25 \%$ and $5 \%$ for distant metastases [10]. In the case of high malignant potential tumours the risk of recurrence and distant metastases is $25 \%$ in each case [10]. It is estimated that local recurrence does not worsen the prognosis [10].

On ultrasound examination, phyllodestumour is a polycyclic lesion of mixed echogenicity in which fluid spaces are present. Its structure bears resemblance to the crosssection of a cabbage. On mammography examination, the neoplasm is usually observed as shadowing with blurred contours and polycyclic structures. Most frequently it is not a spicular lesion. Microscopic verification of the neoplasm is conducted with the use of fine-needle aspiration biopsy (BACC), oligobiopsy, mammotome biopsy or vacuumassisted biopsy (VAB). However, these methods have their diagnostic limitations due to the fact that tumours are often

Address for correspondence: Barbara Madej, Breast Surgery Department the Cardinal Rev. Wyszynski Voivodship Specialist Hospital in Lublin Anatomy Department Medical Uniwersity of Lublin, Poland

E-mail: basia.madej@interia.pl

Received: 15 July 2013; accepted: 17 February 2014; first published: December 2016 histologically non-homogenous [11]. Phyllodestumour can be composed of adenofibroma or ductal cancer. Sometimes, various parts of the tumour have different grades of malignant potential [2]. In such cases there is no possibility to obtain diagnostic material with any of the above-mentioned methods, microscopic verification is performed with the use of so-called excisional surgical biopsy [12].

The treatment of choice in the case of phyllodestumor is excision of the tumour together with a $1-2 \mathrm{~cm}$ margin of healthy tissue. However, standardised treatment in the case a of tumour of high malignant potential or lesions affecting the whole breast is mastectomy. In patients with axillary lymph nodes metastases, modified radical mastectomy is recommended $[13,14]$. Fortunately, in the majority of patients, the course of the disease and prognosis are good [13].

\section{CASE DESCRIPTION}

A female Caucasian patient aged 13 was admitted to the Department of Children's Surgery in order to diagnose a significant swelling and enlargement of her right breast. She was a schoolgirl in secondary school and came from a middle income family living in a small Polish village. On the basis of the obtained medical history it was known that the patient herself had found an insignificant thickening in her right breast five months prior to hospitalisation. Within the next two months she had observed a significant growth of the tumour which resulted in striae of the skin. This persuaded the girl's mother to bring her daughter to the family doctor who qualified the tumour present in the breast as being changes typical for the period of puberty. The patient was not directed to any additional examinations or to consultation with a specialist.

Three months later, the right breast was even more enlarged, inappropriately in comparison to her left breast, and the disproportion and asymmetry between breasts was extremely significant. The girl's right breast was more sensitive to touch, significantly enlarged, and of denser consistency in comparison to her left breast. On her right breast there were increasingly more visible striae of the skin. 
Two months later, the patient, after having had her next appointment with a family doctor, was directed to a surgical outpatient clinic. On the basis of physical examination she was instructed to have antibiotic therapy and observation. She then again had a medical consultation in the surgical outpatient clinic. The girl's constantly enlarging breast encouraged the doctors to hospitalise her and the patient was directed to the Department of Children's Surgery.

In the department, she had an ultrasound examination of the breast (USG) and fine-needle aspiration biopsy (BACC) supervised by USG. Cytologic examination found numerous instances of protein mass, the presence of ductal epithelial cells, cells characterised by pleomorphism of the nucleus and presence of tiny intranucleus vacuoles. Microscopic diagnosis indicated mild breast cell proliferation, so-called juvenile cell proliferation. The pathologist suggested further diagnostics and treatment in an endocrinological outpatient clinic. However, the endocrinologist had no suggestions for treatment.

After the patient's stay at the Department of Children's Surgery, she was admitted to an oncologic surgery outpatient clinic, where on the basis of clinical and ultrasound examination of the breast she was diagnosed with phyllodestumour. Repeated fine-needle aspiration biopsy performed in the outpatient clinic confirmed the clinical diagnosis and was the basis for directing the patient to the Breast Surgery Department.

A chest radiograph and abdominal ultrasonography showed no pathological changes. The results of various laboratory tests revealed no abnormalities. Ultrasound examination of the breasts found a well-separated pathological non-homogenous mass, low-echogenic, with scarce signs of vascular flow within the right breast. The lesion affected the breast between 3 - 9 o'clock, occupying about half of the breast. The size of the lesion was estimated at $120 \times 70 \times 35 \mathrm{~mm}$. No pathological changes were seen in the left breast.

The patient was qualified for surgical treatment quadrantectomy of the right breast.

The patient was admitted to the Breast Surgery Department and operated on. Under general anaesthesia, the tumour was reached through an incision in the submammary fold. The lesion was well-separated, filling half of the breast (Fig. 1). The tumour was removed completely with a $2-\mathrm{cm}$ margin

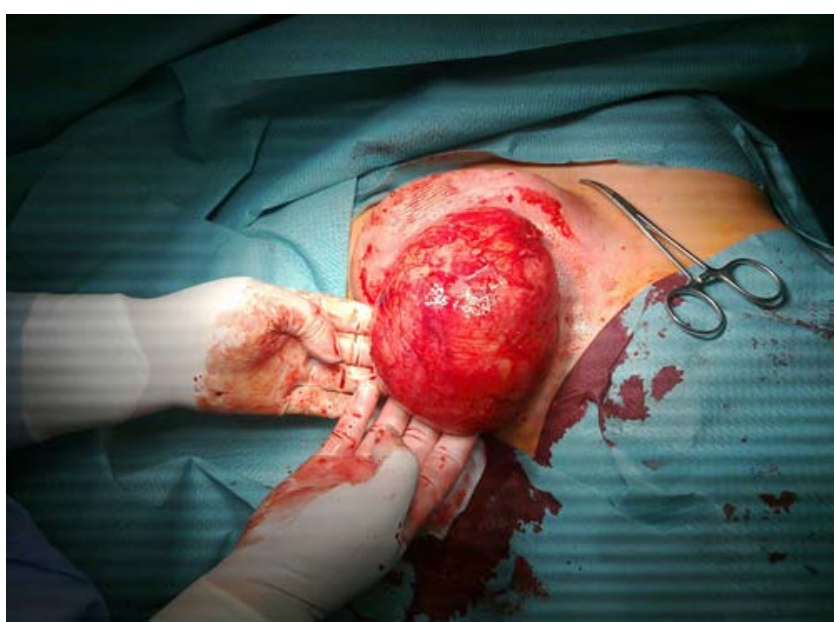

Figure 1. Phyllodes tumour of the right breast in a 13 -year-old patient intraoperative picture of healthy tissues. A reconstructive operation of the nipple and the gland was performed.

There were no complications in the postoperative course. The patient was released home in good condition six days after the operation. On the basis of pathological examination, a final diagnosis was established - phyllodestumour with low malignant potential. The patient remains under constant oncologic observation. There has been no recurrence of the disease to-date. Cosmetic results after the procedure are very good - the shape of the breast is natural and the scar is barely visible (Fig. 2, Fig. 3).

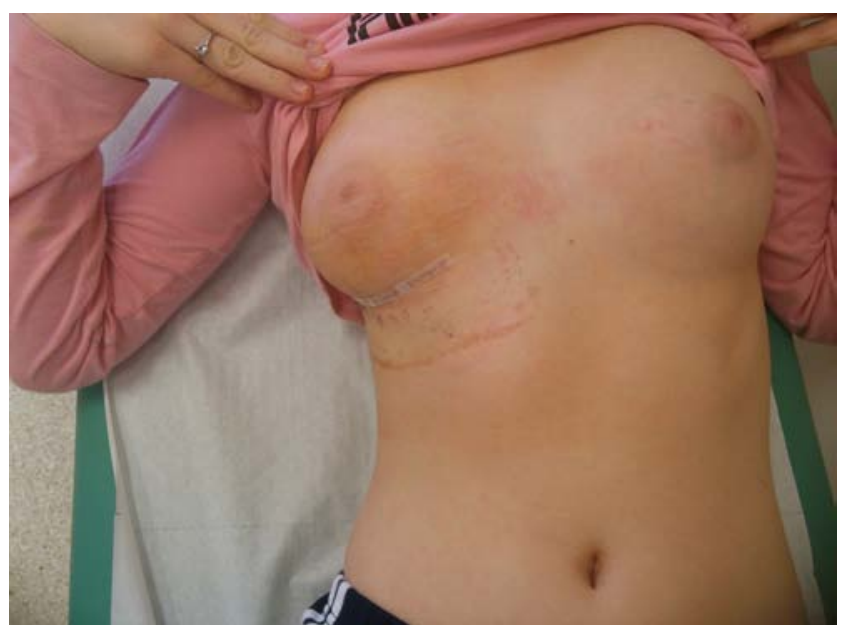

Figure 2. Phyllodes tumour of the right breast in a 13-year-old patient postoperative picture 3 days after the operation

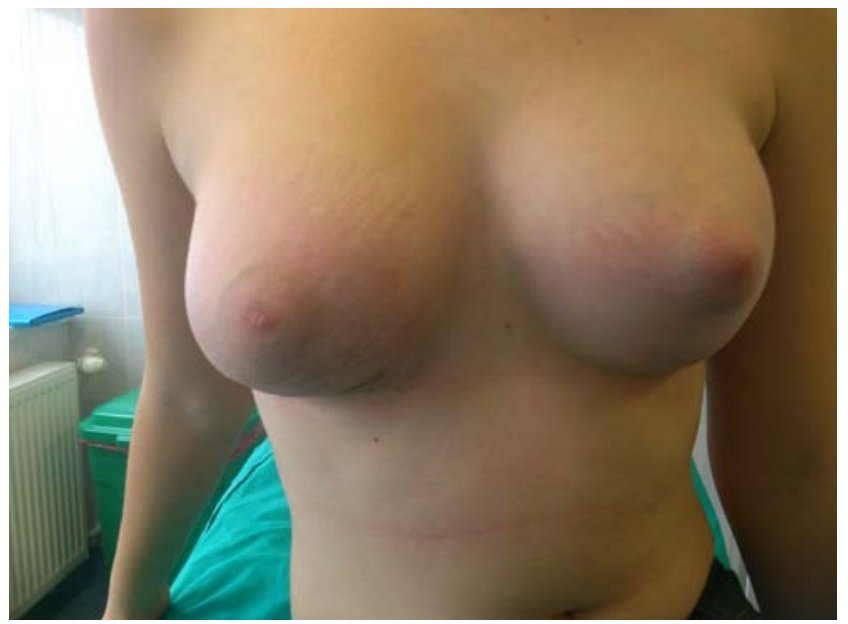

Figure 3. Phyllodes tumour of the right breast in a 13-year-old patient postoperative picture 3 weeks after the operation

\section{DISCUSSION}

The diagnostics of breast diseases in children and teenagers is often conducted inappropriately. The reason for that can be little clinical experience of general practitioners (GPs), pathologists, paediatricians and gynaecologists in that field, as well as the rare incidence of this type of lesion in children. An additional factor making the establishment of diagnosis more difficult is the patient's place of residence. Small towns and villages have no specialist diagnostic facilities to treat breast diseases [15]. Centres for the early detection of cancer and outpatient clinics of breast diseases 
are located in bigger cities. There is no plan for training GPs in this matter. No organisational plan has been devised which would list guidelines where patients suffering from breast diseases should be directed. Neither has it been stated whether it should lie within the competence of oncologists or paediatricians. Maslach et al. [15] showed, that people who live in the rural areas of Poland usually have worse access to health care services and therefore worse access to effective oncological treatment. The authors underlined that especially the situation of young women suffering from breast cancer is the most severe in the group living in rural areas. Moreover, such a group is also characterized by the lowest 5-year survival rate [15].

However, the problem is not insignificant. The incidence of breast cancer in Poland has been on the increase. The disease affects younger and younger women. Genetic investigations conducted on an increasing scale enable determination of a family thread with mutations which are inherited and which predispose to the development of breast cancer. In such cases, even teenagers can suffer from breast cancer. However, such people have some preventive methods at their disposal.

Furthermore, it is also known that some benign lesions diagnosed in teenage years can increase the risk of the incidence of breast cancer in the future. According to GarciaClosas et al. [10], the detection of some benign lesions during adolescence is an additional risk factor for developing breast cancer in subsequent years [10].

Delayed diagnostics and treatment of phyllodestumour always pose a risk to the patient, whereas delayed introduction of appropriate treatment can lead to an increased canceroustransformation risk of the lesion and the need to undergo breast amputation. Such a procedure is performed in teenage patients and it can permanently change their psyche and influence their entire lives. That is why it is extremely important to formulate a diagnostic algorythm concerning breast diseases in children $[16,17]$. The problem is especially difficult in smaller towns. The diagnostics which took far too long and was mismatched in the clinical case described in the presented study of a girl living in a village with only 305 inhabitants confirms that it is vital to take the steps.

\section{REFERENCES}

1. Farria DM, Gorczyca DP, Barsky SH, Sinha S, Bassett LW. Benign phyllodes tumor of the breast: MR imaging features. AJR. 1996; 167: 187-189.

2. Pawlicki J, Król R, Kajor M, Ziaja J. Case of malignant tumour phyllodes converting to fibrosarcoma. Pol Mer Lek. 2007; 22: 215.

3. Chaney AW, Pollack A, McNeese MD, Zagars GK, Pisters PW, Pollock $\mathrm{RE}$, et al. Primary treatment of cystosarcoma phyllodes of the breast. Cancer. 2000; 89: 1502-1511.

4. Guerrero MA, Ballard BR, Grau AM. Malignant phyllodes tumor of the breast review of the literature and case report of stromal overgrowth. Surg Oncol. 2003; 12: 27-37.

5. Josti SC, Sharma DN, Bahadur AK. Cystosarcomap hyllodes: our institutional experience. Australas Radiol. 2003; 47: 434-437.

6. McGregor GI, Kowling MA, Estle FA. Sarcoma and cystsarcoma phyllodes tumors of the breast - a retrospective review of 58 cases. Am J Surg. 1994; 167: 477-480.

7. Barth RJ. Histologic features predict local recurrence after breast conserving therapy of phyllodes tumors. Breast Cancer Res Treat. 1999; 57: 291-295.

8. Kowalski W, Mierzwa T, Grabiec M, Laskowski R, Walentewicz M. Problems in diagnosing breast cancer in women younger than 25 years old. Ginekol Pol. 2006; 77: 376-381.

9. De Roos WK, Kaye P, Dent DM. Factors leading to local recurrence or death after surgical resection of phyllodes tumours of the breast. Br J Surg. 1999; 86, 396-399.

10. Garcia-Closas M, Brinton LA, Lissowska J. Established breast cancer risk factors by clinically important tumor characteristics. Br J Cancer. 2006; 95: 123-6.

11. Klein S. Evaluation of palpable breast masses. Am Fam Physician. 2005; 71: 1731-1738.

12. Gralec A, Czerwionka-Szaflarska M, Zawadzka-Gralec A, Laskowski $\mathrm{R}$, Zegarski W. Diagnostic and therapeutic procedures in adolescents and young adults with changes of the breast. Wspolczesna Onkol. 2010; $14: 372-375$.

13. Asoglu O, Ugurl MM, Blanchard K, Grant CS, Reynolds C, Cha SS et al. Risk factors for recurrence and death after primary surgical treatment of malignant phyllodes tumors. Ann Surg Oncol. 2004; 11: 1011-1017.

14. Kern KA. Diagnostic options in symptomatic breast disease. In: Cameron JL. Current surgical therapy. Mosby, St. Louis 2001. p. 678-686.

15. Maślach D, Krzyżak M, Szpak A, Owoc A, Gębska-Kuczerowska A, Bielska-Lasota M. Differences in results of breast cancer curative treatment between urban/rural female population in Podlaskie Voivodship of Poland before introduction of the National Cancer Control Programme. Ann Agric Environ Med. 2013; 20: 68-71.

16. Al-Mulhim AS, Sultan M, Al-Mulhim FM, Al-Wehedy A, Ali AM, Al-Suwaigh A et al. Accuracy of the "Triple Test” in the diagnosis of palpable breast masses in Saudi females. Ann Saudi Med. 2003; 23: $158-161$.

17. Morris KT, Pommier RF, Morris A, Schmidt WA, Beagle G, Alexander PW et al. Usefulness of the triple test score for palpable breast massesdiscussion, Arch Surg. 2001; 136: 1008-1012. 